\title{
Une communication des organisations comme facteur de protection des risques psychosociaux liés à l'acculturation au numérique (Groupe La Poste)
}

An organization communication as protective factor in psycho-social risks, in relation to digital acculturation (Groupe La Poste)

Émilie Blanc

\section{OpenEdition}

\section{Journals}

Édition électronique

URL : http://journals.openedition.org/communicationorganisation/5268

DOI : 10.4000/communicationorganisation.5268

ISSN : $1775-3546$

\section{Éditeur}

Presses universitaires de Bordeaux

\section{Édition imprimée}

Date de publication : 1 juin 2016

ISBN : 979-10-300-0059-7

ISSN : 1168-5549

\section{Référence électronique}

Émilie Blanc, «Une communication des organisations comme facteur de protection des risques psychosociaux liés à l'acculturation au numérique (Groupe La Poste) », Communication et organisation [En ligne], 49 | 2016, mis en ligne le 01 juin 2019, consulté le 03 janvier 2020. URL : http:// journals.openedition.org/communicationorganisation/5268; DOI : 10.4000/ communicationorganisation.5268 


\section{Une communication des organisations comme facteur de protection des risques psychosociaux liés à l'acculturation au numérique (Groupe La Poste)}

\section{Emilie Blonc' $^{1}$}

Dans le cadre d'une recherche doctorale à La Poste, une recherche-action (RA) est menée sur la prévention des risques psychosociaux (RPS) liés à "l'acculturation au numérique $»^{2}$ des collaborateurs. En effet, le Groupe La Poste et, plus particulièrement, le Centre financier de Toulouse (Groupe La Poste) mettent en place un nouveau plan de communication et d'actions basées sur l'acculturation au numérique qui vise à conduire le changement consécutif à l'essor de la société de l'information et de l'arrivée du numérique, l'objectif étant d'apporter un meilleur service aux clients qui utilisent de plus en plus ces technologies. Le contexte de la recherche est le suivant : une assistante sociale (AS), chercheuse en sciences de l'information et de la communication (SIC) décide de monter un groupe de travail d'AS sur le thème de départ de la RA partant de l'idée que l'acculturation au numérique représente un facteur de RPS pour les collaborateurs. À travers cette RA, nous verrons comment cette acculturation au numérique qui reste un changement organisationnel, ne se révèle finalement pas comme constituant un facteur de RPS chez des postiers qui ont pourtant déjà vécu une première acculturation difficile lors du passage du secteur public au privé. Ce constat nous amènera à interroger le rôle qu'a pu jouer la communication interne de l'organisation en tant quaccompagnatrice de cette acculturation, et comment celle-ci se révèlera finalement constituer un facteur de protection des RPS face à cette acculturation. Ces découvertes nous amèneront également à questionner l'objet même du numérique, car son acculturation semble constituer un changement organisationnel particulier du fait de son utilité en dehors des murs de l'entreprise, ce qui viendra également remettre en question le rôle habituel de la communication des organisations.

\footnotetext{
1 Doctorante en Sciences de l'Information et de la Communication (SIC), Laboratoire Information, Milieu, Médiations (I3M, EA 3820), Université Nice Sophia Antipolis (UNS). Assistante sociale du personnel Centre financier Toulouse- Groupe La Poste ; emilieblanc31@gmail.com

2 http://www.pratiques-rh-au-quotidien.com/2015/04/concevoir-un-dispositif-de-formation-massif-etpersonnalise-aux-opportunites-du-numerique-le-cas-la-poste/
} 


\section{À la recherche d'une prévention primaire des RPS liés à l'acculturation au numérique des postiers}

Comme l'annonce M. Carmès et J. M. Noyer (2013), « Le plissement numérique du monde est en cours et ce processus affecte les socles anthropologiques de nos sociétés " (Carmes, Noyer 2013). Aussi, la transformation numérique engendre des changements tant dans les manières de travailler que dans l'environnement personnel, avec par exemple, l'explosion des réseaux sociaux et le déploiement massif des téléphones mobiles intelligents. Aujourd'hui, la plupart d'entre nous sommes en permanence connectés grâce au web mobile via nos ordinateurs, tablettes et smartphones. Ainsi, cette nouvelle donne numérique oblige-t-elle les entreprises à se transformer pour continuer à satisfaire des clients de plus en plus exigeants, car dans un monde où les échanges s'accélèrent en permanence, les entreprises doivent pouvoir s'adapter rapidement à des situations nouvelles.

Au sein du Groupe La Poste, se pose donc de la même façon, la question des enjeux de cette révolution numérique, car « $100 \%$ des postiers doivent être connectés d'ici $2020 »^{3}$. Le Groupe a donc entamé la transformation de ses activités en développant des solutions digitales et en octroyant les outils modernes aux collaborateurs, afin qu'ils deviennent appétents au numérique et gagnent ainsi en efficacité. Pour définir les dispositifs numériques, nous reprenons la définition proposée par Daniel Peraya (1999) sur le « système socio-technique » qui est " ...une construction au sens technique mais aussi social, puisqu'il matérialise les ambitions, les volontés et les stratégies des acteurs qui l'ont conçu et promu ....au-delà de la matérialité technique... » ou « ...un ensemble de moyens mis au service d'une stratégie, d'une action finalisée, planifiée visant à l'obtention d'un résultat... un dispositif est une instance, un lieu social d'interaction et de coopération possédant ses intentions, son fonctionnement matériel et symbolique, enfin, ses modes d'interaction propres »(Peraya 1999, cité dans Paquienséguy 2006 : 3). Par ailleurs, au sein du Groupe La Poste, et au-delà de la mise à disposition de dispositifs numériques, cette transformation passe également par un plan d'actions visant une acculturation au numérique des postiers. L'objectif est de diffuser une culture numérique à destination de l'ensemble des collaborateurs afin de dédramatiser l'usage des innovations technologiques pour au final, pouvoir mieux conseiller les clients. À l'origine, le terme d'acculturation a été proposé en 1880 par des anthropologues américains. En psychologie sociale, il a souvent désigné « le processus d'apprentissage par lequel un enfant reçoit la culture de l'ethnie ou du milieu auquel il appartient »(Redfield, Linton, Herskovits 1936). En anthropologie culturelle, c'est "l'ensemble des phénomènes résultant du contact direct et continu entre des groupes d'individus de cultures différentes

3 Sylvie Joseph, directrice de la transformation interne au sein de la branche numérique. 
avec des changements subséquents dans les types de culture originaux de l'un ou des autres groupes " (Bastide 1998).

L'acculturation au numérique des postiers représente donc bien un changement organisationnel, et comme tous changements en entreprise, ceux-ci ont des impacts sur le plan psychosocial.

Par exemple, La Poste a déjà subi une première acculturation délicate lorsqu'elle a subi une « greffe culturelle "(Teissier 1963) lors de son passage du secteur public au privé qui a eu de nombreuses conséquences sur les postiers. D'une culture du service public en faveur de l'usager, ils ont dû s'adapter aux règles d'une gouvernance dédiée à l'amélioration de la rentabilité. Ainsi, ont-ils dû adopter une logique de compétitivité à destination du client. Pour certains postiers, ces transformations ont été synonymes de dégradation de leurs conditions de travail. Une vague de suicides, de souffrance au travail, d'épuisement professionnel et de mal-être a fait son apparition. Ces phénomènes peuvent être identifiés comme des risques psychosociaux, bien que le terme risque psychosocial (RPS) soit le plus souvent associé à la notion de stress.

Selon le rapport BIT stress $2000^{4}$, il existe trois niveaux d'actions complémentaires de prévention des RPS. La prévention tertiaire permet de prendre en charge les individus affectés par le stress. Le plus souvent, il s'agit du rôle quotidien du médecin du travail ou de l'assistante sociale. La prévention secondaire aide les individus à développer des capacités afin de faire face au stress, par exemple, par la formation ou des techniques de développement personnel. Enfin, la prévention primaire permet d'éliminer ou de réduire les sources de stress, bien en amont, par exemple lors d'un changement organisationnel. Elle s'effectue grâce à un pré-diagnostic qui implique les travailleurs eux-mêmes, le but étant de produire une analyse de leur situation de travail, notamment en identifiant des facteurs de risque et de protection des RPS.

Selon le rapport Gollac ${ }^{5}$, les principaux facteurs de risque psychosocial et donc par là même, de protection, peuvent être classés en six thèmes : l'intensité du travail et le temps de travail, les exigences émotionnelles, l'autonomie, la qualité des rapports sociaux au travail, les conflits de valeurs, et enfin, l'insécurité de la situation de travail.

Ainsi, afin de mener des actions dans le cadre d'une prévention primaire des RPS, l'AS, chercheuse en SIC décide de créer un groupe de travail réunissant plusieurs AS et collaborateurs des services financiers (SF) de La Poste sur la prévention des RPS liés à l'acculturation au numérique des collaborateurs, thème de départ de la recherche-action (RA). En effet, la RA est une démarche pertinente " pour développer des stratégies permettant de soutenir des professionnels attachés au développement de leur métier » (Meyer 2006). Dans la démarche de RA proposée par Kurt Lewin (1890-1947) à qui il est

4 http://www.ilo.org/global/about-the-ilo/media-centre/press-releases/WCMS_008282/lang--fr/index.htm 5 www.travailler-mieux.gouv.fr/Mesurer-les-facteurs-psychosociaux.html 
généralement attribué le concept dans le cadre de la psychologie sociale, « le chercheur se transforme en formateur et en agent de changement ». La RA semble donc être une des méthodes les plus adaptées à une réflexion collective par et pour les collaborateurs, afin d'accompagner la conduite du changement et d'agir sur la prévention des risques psychosociaux.

Pourtant, à l'issue de leurs observations et enquêtes de terrain, un point particulier attire l'attention des AS. En effet, les professionnels se rendent compte que l'hypothèse de départ, à savoir : l'acculturation au numérique est un facteur de risque psychosocial pour les postiers, ne semble finalement pas en représenter un à ce stade. Il apparaît donc intéressant d'étudier le plan d'action mis en place par la communication interne, et notamment celui du Centre financier de Toulouse, qui a veillé à accompagner cette acculturation au numérique.

\section{Une communication des organisations au format et au déroulé innovants: étude de cas des "petits déjeuners numériques"}

À La Poste, comme habituellement dans les organisations, la diffusion de la culture de l'organisation est assurée par la direction de la communication qui a pour mission de produire un discours donnant du sens, "voire à faire agir dans le même sens »(Parrini-Alemanno, Parent 2013 : 249),(Elise Maas 2013). En effet, la communication interne doit développer la dynamique collective, et faire vivre et évoluer la culture interne. Ainsi, tout changement organisationnel implique un changement culturel et c'est donc bien par la communication interne de lorganisation que la conduite du changement s'opère. À La Poste, le changement organisationnel est ici représenté par l'arrivée du numérique, et la conduite de ce changement s'opère par l'intégration d'une nouvelle culture, c'est l'acculturation au numérique.

Les «petits-déjeuners numériques»du Centre financier de Toulouse s'insèrent dans un plan de communication et d'actions plus large et ils sont intéressants à étudier du fait de l'innovation qu'ils représentent. L'innovation se distingue de l'invention (Schumpeter 1942) car elle relève de la logique du marché ou de l'usage social, contrairement à l'invention qui relève de la logique de la découverte. L'usage est ici défini comme une pratique (Jouët 1993). Les " petits-déjeuners numériques " se présentent donc comme des ateliers mensuels pratiques du numérique. Ils sont des ateliers conviviaux organisés par le service communication afin d'améliorer les connaissances des collaborateurs et ainsi mieux appréhender l'usage des outils digitaux. Au total, dix thématiques ont été abordées en 2014, par exemple, sur Facebook, l'échange de données volumineuses, Twitter, les réseaux sociaux professionnels, ou encore You tube. Quatre mois après le lancement des ateliers, " les petits-déjeuners numériques » ont accueilli plus de 100 participants et ont continué de rencontrer un vif succès au cours des mois suivants. Ces ateliers pédagogiques ont donc été poursuivis sur toute l'année 2015. 


\section{Une communication des organisations comme facteur de protection}

Habituellement, un des objectifs majeurs de la communication interne (Michon 1994) est de faire adhérer l'ensemble des collaborateurs au projet de l'entreprise, et de les faire se comporter selon la charte des valeurs de celle-ci, autrement dit selon une culture d'entreprise prédéterminée. Dans cette perspective, la culture managériale cherche à rationaliser les dimensions humaines des organisations, et vise la performance des collaborateurs.

Le terme de collaborateur s'inscrit dans les pratiques discursives structurant la gestion (Boussard 2008 : 17). Il est progressivement apparu dans les années 1990 et désigne à la fois l'effectif global d'une organisation, les individus membres d'une équipe de travail, et le type de relations entretenues par ceux-ci. Ainsi, la prospérité générale et l'intérêt individuel de chacun seraient intégrés dans une logique commune constituant le terreau contemporain des théories du management. En effet, des éléments de solidarité organique et même d'affectivité sont réintroduits dans les organisations, qui deviennent idéales, car elles seraient dorénavant composées de collaborateurs impliqués et dévoués, se coordonnant de manière fluide et spontanée, où chaque individu deviendrait un rouage consciencieux et actif d'une même entité. Pourtant, « le collaborateur tel qu'il est postulé [...] résulte d'une tentative de formatage symbolique afin de favoriser l'intégration de l'individu au travail dans lorganisation idéale et rationalisée » (Bouillon, Maas 2009). Le but est de le convaincre de mettre à disposition de lorganisation l'ensemble de ses capacités personnelles en lui demandant un investissement global car l'organisation ne s'intéresse plus uniquement aux savoir-faire, mais également aux personnalités.

Pour ce faire, l'instrumentalisation de la communication (Winkin 2003) en est un moyen courant, car les politiques de communication possèdent une dimension communicationnelle idéologique (Bouillon 2008 ; Bouillon, Bourdin, Loneux 2007). Ce type de culture, " construit dans un objectif fonctionnaliste (Thévenet 2003) “...” est souvent considéré comme un message top down, et manipulateur, par les collaborateurs (Maas 2011) " (ParriniAlemanno, Parent 2013 : 250). En effet, le plus souvent, ceux-ci ne sont pas dupes des stratégies en présence pour les construire comme des acteurs d'une organisation idéale, et perçoivent le décalage entre les constructions discursives constitutives de la stratégie de communication qu'ils peuvent caractériser par exemple comme " langue de bois ", " top down ", " corporate » et leur réalité quotidienne. Ainsi, les collaborateurs n'adhèrent-ils pas forcément toujours à la culture de leur entreprise, et à plus forte raison en cas de changement organisationnel où des freins et résistances à la coopération peuvent émerger.

Par conséquent, tout laisserait à croire que l'acculturation au numérique des postiers rencontrerait des difficultés : elle représente un changement organisationnel et à ce titre peut générer des RPS d'autant plus que les 
postiers ont une moyenne d'âge de cinquante ans (donc ils sont d'une génération nétant pas née avec les nouvelles technologies), et d'autant plus qu'ils ont déjà subi une première acculturation difficile lors du passage du secteur public au secteur privé. En effet, ces fonctionnaires d'État possédaient une culture professionnelle forte et presque à l'opposé des valeurs de la culture d'entreprise d'aujourd'hui (compétitivité, rentabilité, ...). Par certains aspects, ils gardent donc leur propre culture qui ne correspond pas forcément à la culture véhiculée par les outils de communication. Il y aurait donc fort à parier que ces « collaborateurs forcés " aient d'autant plus une vision critique de cet acculturation au numérique imposée par la direction des opérations et donc par la communication interne puisque celle-ci pourrait être perçue, d'une manière générale par les collaborateurs, comme un instrument visant uniquement à réaliser les objectifs de l'entreprise.

Pourtant, force est de constater que " les petits-déjeuners numériques » ont rencontré un vif succès et que les $\mathrm{AS}$ ont pu constater à ce stade que l'acculturation au numérique ne représentait pas pour la majorité des collaborateurs un facteur de RPS. Des pistes de compréhension de cette découverte inattendue vont donc être explorées.

En effet, il semblerait que ce format innovant fonctionne car il offre un espace d'expérimentation, d'échanges et de discussion aux collaborateurs qui fait culture au sens où celle-ci est le produit des interactions (Winkin, 2001) et où elle devient une « culture en action (Maas, 2013)». Dans cette perspective, l'organisation n'a pas une culture, celle qui est habituellement instrumentalisée par les discours managériaux et par la communication interne, mais elle est une culture en train de se faire, dans le sens où elle devient une réalité de la culture organisationnelle structurant les collectifs dans l'action. En effet, ici, les collaborateurs participent de cette culture au sein de ces espaces de médiation que sont les "petits-déjeuners numériques " qui constituent des moyens aux interactions de s'accomplir. Ici, la communication interne permet donc de créer des collectifs constitués d'individus qui interagissent et qui donc construisent leur propre culture numérique, une culture singulière. En effet, « il apparaît qu'une culture, processus social complexe et dynamique, ne se décrète pas, ne se cascade pas de haut en bas de l'organisation, mais quelle émerge d'une construction des acteurs (Maas, 2013) " (Parrini-Alemanno, Parent 2013 : 255). Elle s'acquiert par les interactions et constitue un langage commun. Elle lie les individus au sein d'un même groupe partageant par exemple les mêmes connaissances, et se compose de normes et rites construits par le collectif. En effet, dans les années 2000, et pour Giorgio Agamben, les technologies de l'information et de la communication ont un effet sur leurs utilisateurs, et inversement : "Au développement infini des dispositifs de notre temps, correspond le développement tout aussi infini des processus de subjectivation " (Agamben 2007). Pour cet auteur, lorsque les technologies sont imposées au travail, et pour qu'elles se fassent accepter, les usagers se 
les réapproprient, en fonction du contexte, par plusieurs processus de subjectivation. Ainsi, en les contournant ou en inventant de nouveaux usages, ils gagnent en liberté.

De même, pour Edgar H. Schein, lorganisation est une culture non fabriquée. Il propose d'arrêter de voir la culture en tant qu'objet. Il pense qu'il est nécessaire de pouvoir bien communiquer en allant au-delà des frontières culturelles et en visant la compréhension mutuelle. Autrement dit, il insiste sur l'utilité d'apprendre à communiquer par un véritable dialogue qui nécessite écoute et réflexivité. Hatch enrichie le modèle de culture organisationnelle proposée par Schein avec la perspective interprétative et symbolique en considérant la culture non plus de manière statique mais en tant que dynamique ou processus en mouvement. Par ailleurs, pour Weick $(1985,1979)$, c'est la culture qui donne du sens aux actions, donc l'action précède la réflexion et le sens donné. Dans ce cas, la culture agit comme un contexte qui facilite l'interprétation d'une situation en révélant le sens à retenir. C'est l'explication privilégiée qui émane au fil des conversations. Les clés de compréhension de lorganisation sont ensuite diffusées dans le discours, par exemple, lors des échanges ayant lieu entre les membres de l'organisation.

Par conséquent, l'innovation des "petits-déjeuners numériques » en permettant aux collaborateurs d'avoir un espace de médiations, par l'expérimentation et les interrelations qu'ils permettent entre les membres, permet également à ces derniers de créer leur propre culture du numérique. Ainsi, grâce à l'adhésion et à la participation que ces formats pédagogiques suscitent, « les petits-déjeuners numériques » constituent (et par là même la communication interne organisationnelle) des facteurs de protection des RPS de l'acculturation au numérique, d'autant plus que l'objet que constitue le numérique est un objet singulier qui transcende l'organisation.

\section{Une communication des organisations qui favorise la citoyenneté?}

A priori, la communication des organisations porteuse de la stratégie managériale et instrument d'adhésion aux valeurs de l'entreprise doit s'arrêter aux frontières de celle-ci. Or, le dispositif numérique a ceci de particulier qu'il compte des caractéristiques spécifiques semblant donner à la communication des organisations et malgré elle, un nouveau rôle.

En effet, ici, l'objet numérique ne semble pas être considéré par les collaborateurs comme un nouveau changement organisationnel à ingurgiter, mais semble faire culture, et être un élément de la participation et de l'adhésion des collaborateurs. À ce titre, il représente un objet différent et particulier car il semble faire sens pour les collaborateurs d'une autre manière.

Les technologies bouleversent les modes de vie, les façons de travailler, et d'être ensemble. Par exemple, ces modifications sont l'individualisation des relations, la mutation des identités professionnelles et sociales, la reconfiguration des cultures, ou encore la transformation des représentations et des 
pratiques sociales dans les organisations. Le numérique est donc partout et entraîne avec lui de nombreux effets.

Par exemple, l'anthropologue Or Broadbent a travaillé sur les usages personnels des TIC au sein des institutions. Son approche pluridisciplinaire permet d'appréhender les communications privées par les TIC dans leur complexité : les normes sociales (sociologie), les émotions (psychologie clinique), et les processus cognitifs (ergonomie cognitive), afin de comprendre le réel de la vie numérique des individus. Cette vie s'exprime à travers différents canaux de communication à savoir le téléphone (fixe et portable), les sms, la messagerie électronique, la discussion instantanée, Skype, Facebook... L'objectif est de voir comment les TIC sont utilisées à des fins personnelles. Dans quel but ? À quel moment de la journée ? Pour communiquer avec qui ? Au final, il est démontré que les TIC, dans leur utilisation personnelle, sont employées par les individus pour communiquer avec un cercle de personnes proches, en moyenne cinq (famille et/ou amis). Ainsi, «à la lumière de la théorie de l'attachement (Bowlby 1940), Stefana Broadbent explique l'effet de ces communications numériques privées sur les individus : « la proximité physique et psychologique avec les proches permet de diminuer l'anxiété, le stress, et par là même d'augmenter le sentiment de sécurité et le bien-être psychologique " (Broadbent 2011). Ce type de communications privées, appelé le "grooming " (Ling 2004), et notamment les communications textuelles, sont en hausse car il est plus facile d'échanger du texte (mails, sms), car le texte isole en toute discrétion de l'environnement immédiat. Ainsi, les communications digitales personnelles permettraient d'actualiser l'équilibre émotionnel des individus, alors que jusqu'à l'apparition des TIC, la séparation stricte des sphères privées et professionnelles était fortement revendiquée.

Par conséquent, cette utilisation massive des nouvelles technologies et cette nécessité à le faire dépassent les frontières de l'entreprise. On peut donc se demander si cet engouement pour l'acculturation au numérique des postiers, alors qu'elle aurait pu constituer un facteur de RPS, n'a pas réussi par le fait même que l'acculturation au numérique devient une nécessité pour tous et trouve une utilité en dehors des frontières de l'entreprise et dans sa vie quotidienne.

En effet, ici, l'acculturation au numérique ne semble pas être considérée par les postiers comme un nouveau changement organisationnel auquel il faut adhérer, car son utilité dépasse l'entreprise. Cette acculturation possède une utilité au cœur même de la vie quotidienne des individus : que ce soit sur leur temps de travail, par exemple, pour être en relation avec leurs proches, qu'une fois sortis de l'entreprise, par exemple, pour faire des démarches administratives (déclarer ses impôts, faire ses courses via le drive, etc...). Le numérique a donc ceci de particulier qu'il est un objet servant les intérêts de l'entreprise mais aussi ceux des collaborateurs. À ce titre, l'acculturation au numérique 
constitue un changement organisationnel original agissant à la fois dans la vie professionnelle et dans la vie privée des collaborateurs.

Qu'en est-il donc de la communication des organisations qui véhicule ce changement? On peut considérer que ses actions ont une portée en dehors des murs de l'entreprise sans que ce soit le but premier recherché au départ (qui reste l'atteinte des objectifs de l'entreprise). En effet, dans cette perspective, ne pouvons-nous pas considérer que cette communication des organisations acquiert un rôle civique en réduisant les inégalités d'accès au numérique ? Ce qui conférerait par ailleurs à la communication des organisations un nouveau rôle et ouvrirait par là même de nouvelles voies de recherche dans le domaine...

L'acculturation au numérique en tant que changement organisationnel aurait pu générer des RIS dans cette organisation déjà fragilisée par de multiples réorganisations et une première acculturation vécue non sans difficultés par les postiers. Pourtant, le service communication a pu impulser de véritables innovations, notamment avec les «petits déjeuners numériques » qui ont permis d'accompagner les collaborateurs dans cette appropriation du changement organisationnel, conférant par là même à la communication de l'organisation de nouvelles fonctions. En effet, à travers cette étude, c'est le rôle de la communication des organisations qui se trouve finalement questionnée car celle-ci apparaît comme un facteur de protection des RPS liés à l'acculturation au numérique. De même, l'objet même du numérique étant complexe et encore en pleine définition, celui-ci semble conférer à la communication des organisations un nouveau rôle hors des murs de l'entreprise et dans le même temps, une dimension plus large au sein de la société.

\section{BIBLIOGRAPHIE}

http://www.pratiques-rh-au-quotidien.com/2015/04/concevoir-un-dispositif-deformation-massif-et-personnalise-aux-opportunites-du-numerique-le-cas-la-poste/

http://www.ilo.org/global/about-the-ilo/media-centre/press-releases/ WCMS_008282/lang--fr/index.htm

www.travailler-mieux.gouv.fr/Mesurer-les-facteurs-psychosociaux.html

AGAMBEN G., "Qu'est-ce qu'un dispositif ? ", Rivages poche, petite bibliothèque, 2011, $1^{\text {re }}$ édition en italien 2007, p. 30-31.

ARASZKIEWIEZ J., « Savoir, pouvoir, sujet : De la domestication de l'être au monde sans couture des technologies de l'information et de la communication ", Les cabiers de la $S F S I C n^{\circ} 10$, juin 2014, p. 141-147.

BASTIDE R., " Acculturation ", Encyclopedia Universalis, 1998, p. 1-114 c et suivant.

BOUILLON J. L., MAAS E., Figures de l'individu au travail, figures du « collaborateur », Communication E organisation, n ${ }^{\circ} 36,2009$, p. 56-68. 
BROADBENT S., L'intimité au travail. La vie privée et les communications personnelles dans l'entreprise, Limoges, FYP éditions, 2011,192 p.

CARMES M., NOYER J. M. (dir.), Les débats du numérique, Paris, Presses des Mines, coll. Territoires numériques, 2013, 279 p.

COULON A., L'école de Chicago, Paris, Presses Universitaires de France, coll. Que sais-je ? 2012, 126 p.

DUBOIS J., MITTERAND H., DAUZAT A., Dictionnaire d'étymologie, Evreux, Larousse, 2001, 864 p.

FERREOL G., Dictionnaire de sociologie, Paris, A. Colin, 2011, 329 p.

GROSJEAN S., BONNEVILLE L., La communication organisationnelle. Approches, processus et enjeu, Québec, Chenelière Education, 2011, 346 p.

MEYER V., De l'utilité des recherches-actions en SIC, Communication Ė organisation, $n^{\circ} 30,2006$, p. 89-108.

MICHON C., Management et communication interne : les six dimensions qu'il faut considerer, Communication E'organisation, n 5, 1994.

PARRINI-ALEMANNO S., dir, Communication organisationnelle, management numérique, Paris, L'harmattan, 2014, 446 p.

PARRINI ALEMANNO S., PARENT B., dir, Les communications organisationnelles. Comprendre, construire, observer, Paris, L'harmattan, 2013, 315 p.

PYBOURDIN I, GRANGER L., "Engagement et réseau : une expérience de recherche-action REVE », Les cabiers de la SFSIC n 10, Juin 2014, p. 161-162.

REDFIELD R., LINTON R., HERSKOVITS M.J., Memorandum on the study of acculturation, American Anthropology, n ${ }^{\circ}$ 38, 1936.

STOETZEL J., La psychologie sociale, Paris, Flammarion, 1963, 317 p.

TEISSIER C., La Poste : logique commerciale / logique de service public. La greffe culturelle, Paris, L'harmattan, 1963, 303 p.

VALLERY G., LEDUC S., Les risques psychosociaux, Paris, Presses Universitaires de France, 2012, 126 p.

Résumé : Dans une recherche-action menée au sein de La Poste, nous problématisons le rôle d'une «communication organisante» dans la prévention des risques psychosociaux liés à un changement : « l'acculturation au numérique». À l'issue de nos recherches, il apparaît qu'une majorité des collaborateurs n'est pas en difficulté dans ses usages du numérique, compte tenu des actions d'accompagnement à l'acculturation mises en place par la direction de la communication, notamment les "petits déjeuners numériques». La communication des organisations se révèle donc être un facteur de protection des RPS de cette «acculturation au numérique ", et vient par là même questionner son rôle en tant que contributrice de la citoyenneté.

Mots-clés : acculturation au numérique, dispositifs numériques, prévention des risques psychosociaux (RPS), recherche action (RA), communication des organisations. 
Abstract : This text deals with digital literacy at the French Post Office Company. On this occasion, that was an opportunity for a social worker to join a working group and study with an action-research the consequences for workers on their mental suffering. Besides after considering the links between psychological risks and digital literacy, he showed that it was not true for the postal workers. Indeed the actions that had been put in place in the company were effective help and much more, that the communication of organisations is a protection against psychological risks and social exclusion.

Keywords : digital literacy, digital technologies, psychological risks prevention, actionresearch, communication of organization. 